\title{
The feasibility of automated online flow cytometry for in-situ monitoring of microbial dynamics in aquatic ecosystems
}

\author{
Michael D. Besmer ${ }^{1,2}$, David G. Weissbrodt ${ }^{1,3}$, Bradley E. Kratochvil' ${ }^{1}$, Jürg A. Sigrist ${ }^{1}$, \\ Mathias S. Weyland ${ }^{1}$ and Frederik Hammes ${ }^{1 *}$
}

\footnotetext{
' Department of Environmental Microbiology, Eawag - Swiss Federal Institute for Aquatic Science and Technology, Dübendorf, Switzerland

${ }^{2}$ Department of Environmental Systems Science, Institute of Biogeochemistry and Pollutant Dynamics, ETH Zürich, Zürich, Switzerland

${ }^{3}$ Institute of Environmental Engineering, Chair of Process Engineering in Urban Water Management, ETH Zürich, Zürich, Switzerland
}

\section{Edited by:}

Susann Müller, Helmholtz Centre for

Environmental Research, Germany

\section{Reviewed by:}

Lee-Ann H. Allen, University of

lowa, USA

Andreas Nocker, Cranfield

University, UK

Andreas H. Farnleitner, Vienna

University of Technology, Austria

Susanne Günther, Helmholtz Centre

for Environmental Research,

Germany

\section{*Correspondence:}

Frederik Hammes, Drinking Water

Microbiology Group, Department of

Environmental Microbiology,

Eawag - Swiss Federal Institute for

Aquatic Science and Technology,

Überlandstrasse 133, 8600

Dübendorf, Switzerland

e-mail: frederik.hammes@eawag.ch
Fluorescent staining coupled with flow cytometry (FCM) is often used for the monitoring, quantification and characterization of bacteria in engineered and environmental aquatic ecosystems including seawater, freshwater, drinking water, wastewater, and industrial bioreactors. However, infrequent grab sampling hampers accurate characterization and subsequent understanding of microbial dynamics in all of these ecosystems. A logic technological progression is high throughput and full automation of the sampling, staining, measurement, and data analysis steps. Here we assess the feasibility and applicability of automated FCM by means of actual data sets produced with prototype instrumentation. As proof-of-concept we demonstrate examples of microbial dynamics in (i) flowing tap water from a municipal drinking water supply network and (ii) river water from a small creek subject to two rainfall events. In both cases, automated measurements were done at 15-min intervals during 12-14 consecutive days, yielding more than 1000 individual data points for each ecosystem. The extensive data sets derived from the automated measurements allowed for the establishment of baseline data for each ecosystem, as well as for the recognition of daily variations and specific events that would most likely be missed (or miss-characterized) by infrequent sampling. In addition, the online FCM data from the river water was combined and correlated with online measurements of abiotic parameters, showing considerable potential for a better understanding of cause-and-effect relationships in aquatic ecosystems. Although several challenges remain, the successful operation of an automated online FCM system and the basic interpretation of the resulting data sets represent a breakthrough toward the eventual establishment of fully automated online microbiological monitoring technologies.

\section{Keywords: flow cytometry (FCM), bacteria, drinking water, monitoring, sensors, automation, online, staining}

\section{INTRODUCTION}

Monitoring microbial dynamics in engineered and environmental aquatic ecosystems is a key step toward a better understanding of the driving forces and consequences of changes in bacterial concentrations and community composition (Stadler et al., 2008; Read et al., 2011). Such ecosystems are often highly dynamic. For instance, engineered ecosystems such as drinking water production and wastewater treatment plants follow daily routines in operation, may suffer from short-term malfunctioning, and are exposed to dynamic changes in raw water composition (Gunther et al., 2012; Hwang et al., 2012). Environmental ecosystems such as oceans, lakes, and groundwater are subject to daily or seasonal variations or sudden events (e.g., acute pollution) (Guadayol et al., 2009; Stadler et al., 2010). Bacterial parameters tend to respond to such variations and events. To establish the links between the microbial dynamics and their causes and implications, accurate and high-throughput measurements of microbial parameters are necessary (Van Den Hoven et al.,
2009). Specifically, in the field of drinking water, an improved understanding of microbial dynamics can support process optimization, microbial risk assessment, and risk management (e.g., Smeets et al., 2010, World Health Organization, 2011). When investigating this type of dynamics, it is crucial to collect data for sufficiently long periods and at high sampling frequencies to fully capture the respective type of pattern (e.g., daily, weekly) (Stadler et al., 2008). This makes optimal monitoring a challenge with respect to the choice of method(s) and practical in-situ implementation.

Only a limited number of microbial detection methods are suitable for monitoring aquatic ecosystems in the detailed manner described above. Major constraints are low sensitivity for changes, low reproducibility, and high demand for labor, time and other resources (Sheikh et al., 2012). In view of these constraints, flow cytometry (FCM) is a promising method for high-throughput routine measurements (Porter et al., 1997; Vives-Rego et al., 2000; Czechowska et al., 2008; Hammes and Egli, 2010) and hence 
has been widely applied in the analysis of ecosystems including seawater (Legendre et al., 2001), freshwater (Gregori et al., 2001), wastewater (Foladori et al., 2010), and drinking water (Vital et al., 2012). Direct FCM measurements are possible for organisms with auto-fluorescence (e.g., phytoplankton) (Pomati et al., 2013) or organisms with fluorescence reporter genes [e.g., green fluorescence protein (GFP)] (Arnoldini et al., 2013). Other microorganisms — such as small, environmental bacteria-need to be stained with a fluorescent dye before measurement in order to distinguish them from background and/or to detect specific cellular features (Prest et al., 2013). In light of the requirements for ecosystem monitoring discussed above, one of the principle challenges is to develop robust, automated FCM systems that can stain and measure over extended time periods (e.g., weeks to months) in short intervals (e.g., 10-15 min).

The concept of FCM automation is not new. The first automated systems with and without staining procedures were developed for medical and biotechnology applications (Omann et al., 1985; Lindberg et al., 1993; Zhao et al., 1999; Abu-Absi et al., 2003). In environmental sciences, automated systems were developed for auto-fluorescent pigments (e.g., phytoplankton) (Dubelaar et al., 1999; Thyssen et al., 2008). More recently systems were described for measuring fluorescence from genetically modified organisms in biotechnology (Broger et al., 2011; Brognaux et al., 2013) and a prototype was developed specifically for drinking water analysis (Hammes et al., 2012). The latter study describes laboratory-scale testing of automated sampling, staining, incubation, and measurement as an interesting technique for monitoring aquatic ecosystems. Full-scale application and implementation of automated FCM, though coming with considerable technological and scientific challenges, offer immense potential. Until now automated FCM studies that include fluorescent staining of cells have been limited to short-term $(<60 \mathrm{~h})$ experiments for industrial processes or research applications. Long-term automated FCM measurements focusing on monitoring environmental ecosystems have not yet been explored and considered in depth.

We developed a new prototype online FCM system capable of meeting these challenges. Here we demonstrate the application of this instrumentation and approach on two aquatic ecosystems (drinking water and river water) of differing microbiological quality and dynamics. The novelties of this study are: (i) insitu analysis of real water samples subject to natural events (e.g., rainfall), (ii) fully automated analysis during extended time periods (days-to-weeks), and (iii) detailed analysis of large data sets with respect to microbiological baselines, patterns, and correlations with abiotic sensor data. In addition we critically discuss the advantages and challenges of online FCM and consider the added value of such large data sets in a variety of applications.

\section{MATERIALS AND METHODS ONLINE FCM SYSTEM}

A fully automated staining robot was constructed based on the principles of a previously described system (Hammes et al., 2012). In short, the system was designed to collect discrete water samples at 15-min intervals from a given source (e.g., flowing tap, flowing river). Upon collection the sample was mixed at a 1:1 ratio with a solution of fluorescent dye [SYBR Green I (Life Technologies, Eugene OR, USA)] diluted in sterile TRIS buffer ( $10 \mathrm{mM}, \mathrm{pH}$ 8.0). The final concentration of SYBR Green I in the sample relative to the stock solution was 1:10,000. A bacteriostatic concentrate solution (BD Accuri, Ann Arbor MI, USA) was added to the dye at $5 \mu \mathrm{L} \mathrm{mL}^{-1}$ to avoid contamination in the stain solution. The sample-dye mixture was subsequently incubated for $10 \mathrm{~min}$ at $40^{\circ} \mathrm{C}$ and afterwards automatically pumped to, and analyzed with, a C6 flow cytometer (BD Accuri, San Jose CA, USA). The C6 flow cytometer measured at a flow rate of $66 \mu \mathrm{L} \mathrm{min}{ }^{-1}$ for $30 \mathrm{~s}$ with a lower threshold on the green fluorescence (FL1-H) set at 1000. All other Accuri C6 flow cytometer specifications, filters, and measured variables were identical to those described in Prest et al. (2013). All tubing, pumping, and mixing chambers were rinsed with nanopure water between sampling. The sampling line was flushed with the sample water prior to every new measurement to avoid contamination from earlier steps and ensure that a fresh sample was collected. A cleaning cycle with hypochlorite ( $1 \%$ active chlorine), detergent (Partec, Goerlitz, Germany), and nanopure water was automatically initiated every $24 \mathrm{~h}$.

\section{ENGINEERED ECOSYSTEM: DRINKING WATER}

Drinking water samples were drawn at 15 -min intervals from a continuously flowing cold water tap $\left(16.0 \pm 0.5^{\circ} \mathrm{C}, 0.5 \mathrm{~L} \mathrm{~min}^{-1}\right)$ in the Eawag laboratory building (Dübendorf, Switzerland) during an experimental period of 14 days. The tap water was nonchlorinated groundwater supplied as drinking water by the local water utility. No other parameters were measured during the course of this experiment, but the same water was described in detail in a previous study (Lautenschlager et al., 2010).

\section{ENVIRONMENTAL ECOSYSTEM: RIVER WATER}

A shallow, oligotrophic river (Chriesbach, Dübendorf, Switzerland) was monitored during 12 consecutive days. Samples were measured with online FCM at 15-min intervals from a sedimentation basin, located in an experimental facility adjacent to the river, into which the river water was continuously pumped (approximate retention time: $30 \mathrm{~min}$ ). The same water was simultaneously fed to a 14 -L-PVC reactor (at $0.9 \mathrm{~L} \mathrm{~min}^{-1}$ ) where four abiotic parameters [temperature ( $\mathrm{T})$, dissolved oxygen (DO), electrical conductivity (EC) and $\mathrm{pH}$ ] were measured with online sensor probes (Table 1 ) once every $10 \mathrm{~s}$ in parallel to the FCM measurements. The DO, EC, and $\mathrm{pH}$ sensors were automatically compensated for temperature changes.

\section{DATA EXTRACTION AND ANALYSIS}

The raw FCM data files were analyzed with custom software that enabled batch processing of the large data sets generated in this study. In short, FCM gates were constructed to separate signals of stained bacterial cells from signals of the background and distinguish between the so-called high (HNA) and low (LNA) nucleic acid content bacterial cells. The gating strategy was based on descriptions provided elsewhere (SLMB, 2012; Prest et al., 2013). All generated data, i.e., total cell concentration (TCC) and LNA content bacteria concentration, were subsequently exported in csv format for final processing in MS Excel. In order to separately analyze regular daily patterns and specific events, subsets 
Table 1 | Overview of measured parameters and employed measurement devices for each water type.

\begin{tabular}{lcclr}
\hline Measured parameters & Tap water & River water & Measurement device & Figure/Table \\
\hline Total cell concentration & $\times$ & $\times$ & Online FCM & Figures 1-6 \\
Percentage of LNA content bacteria & $\times$ & $\times$ & Online FCM & Figures 1-6 \\
Conductivity & & $\times$ & Online sensor & Figures 5, $\mathbf{6}$ \\
Temperature & & $\times$ & Online sensor & Figures 5, $\mathbf{6}$ \\
$\mathrm{pH}$ & & $\times$ & Online sensor & Figures 5, $\mathbf{6}$ \\
Oxygen concentration & $\times$ & Online sensor & Figures 5, $\mathbf{6}$ \\
Rainfall volume & & Weather station [NABEL (FOEN and EMPA)] & Figures 3, 5 \\
\hline
\end{tabular}

were extracted from both data sets by subjective selection. Both the data selection and the reasoning behind it are discussed and illustrated in detail in the results and discussion sections below, in Figures 1, 3, and in Table S1 (Supplementary information). The online sensor data set for abiotic parameters was reduced to one point every $15 \mathrm{~min}$ to match the sampling frequency of the online FCM. Multivariate analyses were computed in R ( R Development Core Team, 2008) according to a numerical ecology methodology analogous to the one developed (Weissbrodt et al., 2014) based on Borcard et al. (2011). In short, pair-wise $x-y$ plots were computed between the average baselines of all FCM and sensor parameters for a first visual observation of any apparent correlations. The pair-wise correlations between the full baselines of all parameters were then quantified by the computation of Pearson's correlation coefficients (PCC, linear relationship) and Spearman's rank correlation coefficients (monotonic relationship) after standardization of the FCM and sensor data sets (see Section 8 in supplementary information). The significance of the correlations was assessed by the computation of $p$-values at $95 \%$ confidence level. The pair-wise coefficients were displayed in a heat map for efficient representation of the gradients in positive and inverse correlations between parameters, and for rapid identification of the pre-dominant correlations. In this heat map, the parameters were reordered by hierarchical clustering using the Ward algorithm (see Section 8 in supplementary information). The additional R packages Vegan (Oksanen et al., 2009), Heatplus (Ploner, 2011), and Heatmap.plus (Day, 2007) were used to these ends.

\section{RESULTS}

\section{ENGINEERED ECOSYSTEM: DRINKING WATER}

The 1300 online FCM measurements of flowing municipal tap water during 14 consecutive days showed overall relatively stable TCC and fluorescence fingerprints [i.e., percentage of LNA content bacteria, Prest et al. (2013)], but also revealed daily fluctuations as well as a discernable event/shift in TCC between days 10 and 14 (Figure 1). In order to establish baseline data for this particular ecosystem, we extracted from the combined data set only the values recorded on weekdays, excluding the weekends and the apparent event (days 1-4, 7-10, 14-15; Figure 1 and Table S1). The resulting data set $(n=674)$ had an average TCC of 108.3 \pm 7.8 cells $\mu \mathrm{L}^{-1}$ and an average percentage of LNA content bacteria of $57.6 \pm 2.0 \%$. These data were pooled and sorted in a 24-h window according to their individual measurement times. From this we calculated averages and standard error of the data points of all days within the same 15-min interval (Figure 2). This combined data revealed a clear daily pattern with respect to TCC (Figure 2A). During nighttime (20:00-08:00), the TCC gradually increased at a rate of approximately 0.8 cells $\mu \mathrm{L}^{-1} \mathrm{~h}^{-1}$, and values were mostly above the overall average. The morning hours (08:00-10:00) displayed the highest bacterial concentrations $\left(119.0 \pm 5.8\right.$ cells $\left.\mu \mathrm{L}^{-1} ; n=55\right)$, followed by a sharp decrease between 10:00 and 12:00, a noticeable peak around noon (12:00-14:00) and the lowest concentrations around 15:00. TCC tended to be below the average during daytime. The percentage of LNA content bacteria showed less daily fluctuation although a higher percentage of LNA content bacteria was observed during daytime compared to nighttime (Figure 2B). Only a weak negative PCC $(-0.34, n=674)$ was observed between the TCC and percentage of LNA. On day 10, a remarkable and sudden shift in TCC occurred, with average concentrations increasing to $145.1 \pm 12.5$ cells $\mu \mathrm{L}^{-1}(n=410)$ and only gradually decreasing again until day 14 . In contrast, the percentage of LNA content bacteria remained largely unaffected by the dramatic shift in cell concentration (Figure 1).

\section{ENVIRONMENTAL ECOSYSTEM: RIVER WATER}

The 1100 online FCM measurements of river water at $15-\mathrm{min}$ intervals during 12 consecutive days showed overall stable TCC but revealed clear daily fluctuations and two very dramatic events (days 2 and 10). These were directly attributed to regional rainfall and were visible in considerable changes to both TCC and the percentage of LNA content bacteria (Figure 3). To assess this in detail, a baseline for this particular ecosystem was established by excluding the two rainfall events (days 1-3 and 10-12, Figure 3 and Table S1) and pooling the remaining data together as described above (Figure 4). For all baseline data combined $(n=740)$, the average TCC was $991.3 \pm 98.2$ cells $\mu \mathrm{L}^{-1}$, and the average percentage of LNA content bacteria was $54.9 \pm 1.6 \%$. Moreover, from the averaged data a clear pattern emerged, with the lowest TCC in the morning hours (09:30-12:30) at $854.1 \pm$ 52.1 cells $\mu \mathrm{L}^{-1}(n=84)$ and the highest concentrations in the afternoon (16:00-18:00) at 1063.1 \pm 83.9 cells $\mu \mathrm{L}^{-1}(n=63)$. During the dry periods (i.e., times without rainfall), the percentage of LNA content bacteria increased when the TCC increased (Figure 3), although the fluctuations were smaller than for the TCC. Relative to the baseline data, the two events (days 1-3 and 10-12; Table S1) were unmistakably clear. Both events were a direct consequence of acute bacterial loads following rainfall (Figure 3). Local weather station information showed a total of 

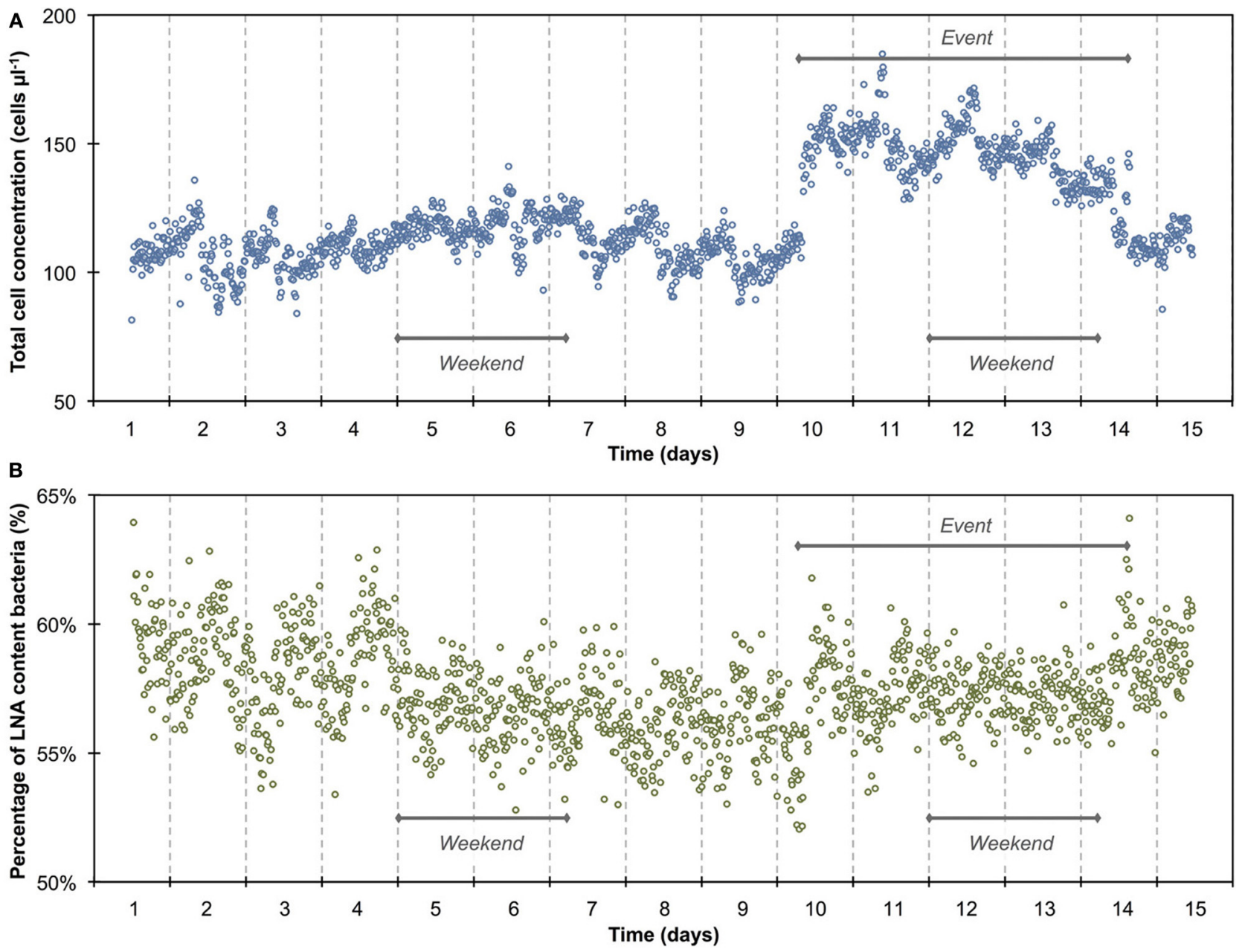

FIGURE 1 | Online FCM measurements of continuously flowing municipal tap water over 14 days, showing the total cell concentration (TCC) (A) and percentage of LNA content bacteria (B) $(n=1302)$. Data points are single measurements at 15-min intervals of water samples stained with SYBR Green I. Daily patterns can be identified for both parameters. In addition, a distinct event with a $50 \%$ increase in TCC can be seen from day 10 to day 14 whereas the percentage of LNA content bacteria was barely affected.
$3.1 \mathrm{~mm}$ of rain falling within $8 \mathrm{~h}$ on day 2 and a total of $1.4 \mathrm{~mm}$ of rain falling within $3 \mathrm{~h}$ on day 10 in the catchment area of the river (Figure S2). As a result, TCC increased above 2000 cells $\mu \mathrm{L}^{-1}$ on day 2 and above 1500 cells $\mu \mathrm{L}^{-1}$ on day 10. During the events, the trend in the percentage of LNA was dissimilar to the dry periods, and values dropped below $45 \%$ during both events as a direct consequence of increased concentrations of HNA content bacteria in the water. Values returned to the baseline within $24 \mathrm{~h}$ after the rainfall ceased. A set of raw FCM data from $48 \mathrm{~h}$ of measurements for the river water experiment is available in video format in the supplementary information (Section 9).

\section{CORRELATIONS WITH ABIOTIC SENSOR DATA}

Online data from four sensors for abiotic parameters complimented the online FCM data with respect to daily pattern analysis and event detection (Figure 5). Figures 5A,B show daily fluctuations in all abiotic parameters. Temperature, DO, and $\mathrm{pH}$ generally peak in the early afternoon and then keep dropping during the night until reaching a low point shortly before noon the following day. In contrast, EC showed a sharp drop in the morning with a low around noon, followed by a sharp increase in the afternoon and a steady increase overnight and into the next morning. The rainfall events were most obvious in the conductivity data (Figure 5B) with other parameters showing comparably little response. In fact, conductivity data correlated remarkably well with TCC data both between and during events (Figure S3). Some sensors also detected apparent weekend-related patterns (notably pH and DO) that were not seen in the FCM data (Figure 5). This was ascribed to wastewater treatment discharge upstream of the sampling point. Hence, for a simplified direct multivariate comparison of all biotic and abiotic data with respect to the daily patterns, we selected only a 4-day period (days 6-9), excluding weekends and rainfall events (Figure 3 and Table S1). The resulting data set $(n=373)$ displayed some interesting relationships between biotic and abiotic parameters (Figure 6). Since the two statistical approaches, i.e., Pearson's linear correlation and 

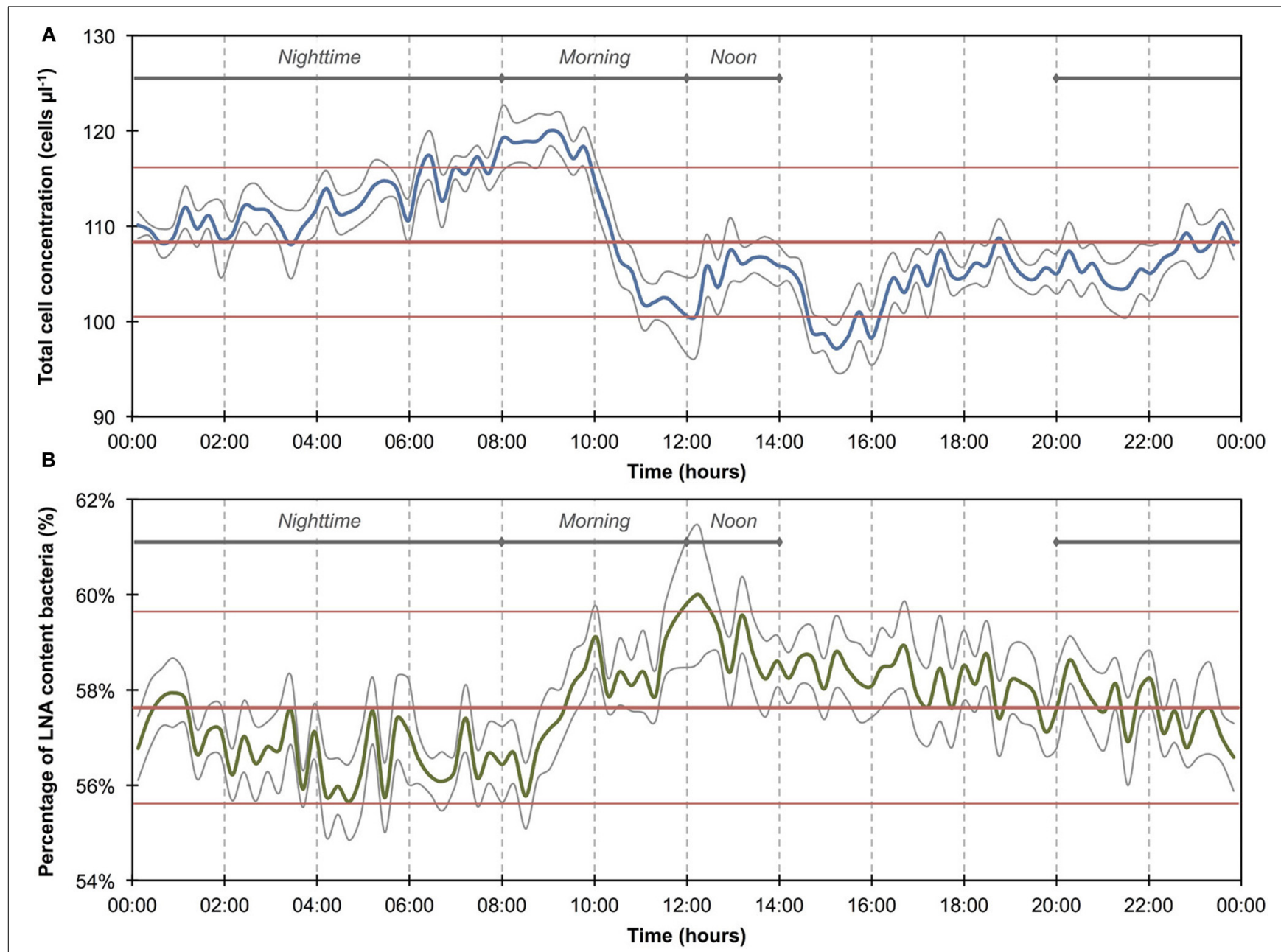

FIGURE 2 | Baseline data of the daily microbiological fluctuations in continuously flowing municipal tap water for total cell concentrations (A) and percentage LNA content bacteria (B). The data set $(n=674)$ comprises only weekday data, excluding weekends and the apparent event (Figure 1). Blue and green lines are 15-min averages of all included days for both parameters. Gray lines represent the standard error for each point of the average. Horizontal red lines show the average (thick line) and standard deviations (thin lines) calculated from all data combined $(n=674)$. A daily fluctuation is clearly detectable in both parameters.
Spearman's rank-order correlation, were providing similar trends, linear relationships between parameters were assumed. Firstly, as suggested above (Figure S3), EC correlated positively with TCC $(\mathrm{PCC}=0.77)$. Secondly, $\mathrm{pH}$ and the percentage LNA content bacteria showed a weaker but significant correlation ( $\mathrm{PCC}=$ 0.45). Thirdly, the dendrogram clustering suggests that EC and $\mathrm{pH}$ were affiliated with TCC and the percentage of LNA content bacteria. Based on the calculated $p$-values computed at $95 \%$ confidence level, the majority of the determined correlations were highly significant. Only weak correlations with low significance were found between the biotic parameters and T and DO. More detailed results for the different parameters are also shown in Figure S4.

\section{DISCUSSION}

Two distinctively different aquatic ecosystems were automatically sampled and measured during multiple days involving minimum human intervention and resulted in FCM data sets in excess of 1000 data points for each experiment. This allowed the establishment of baseline data and the subsequent detection of cyclic patterns, gradual changes, and sudden events for each ecosystem. The automated approach described herein extends the state-of-the-art of in-situ monitoring of microbial dynamics in complex engineered and environmental ecosystems considerably. It furthermore allows for a meaningful combination of microbial data with widely applied online sensor data of abiotic parameters.

\section{BACTERIAL DYNAMICS IN DRINKING WATER}

The average TCC measured in this study corresponded to typical values for non-chlorinated drinking water (Hammes et al., 2008; Lautenschlager et al., 2010; Vital et al., 2012). We observed a reproducible daily pattern in the data (Figures 1, 2) as well as an unexplained dramatic event in the second week of measurements. To our knowledge, no previous studies investigated tap water at such high temporal resolution, and therefore the 

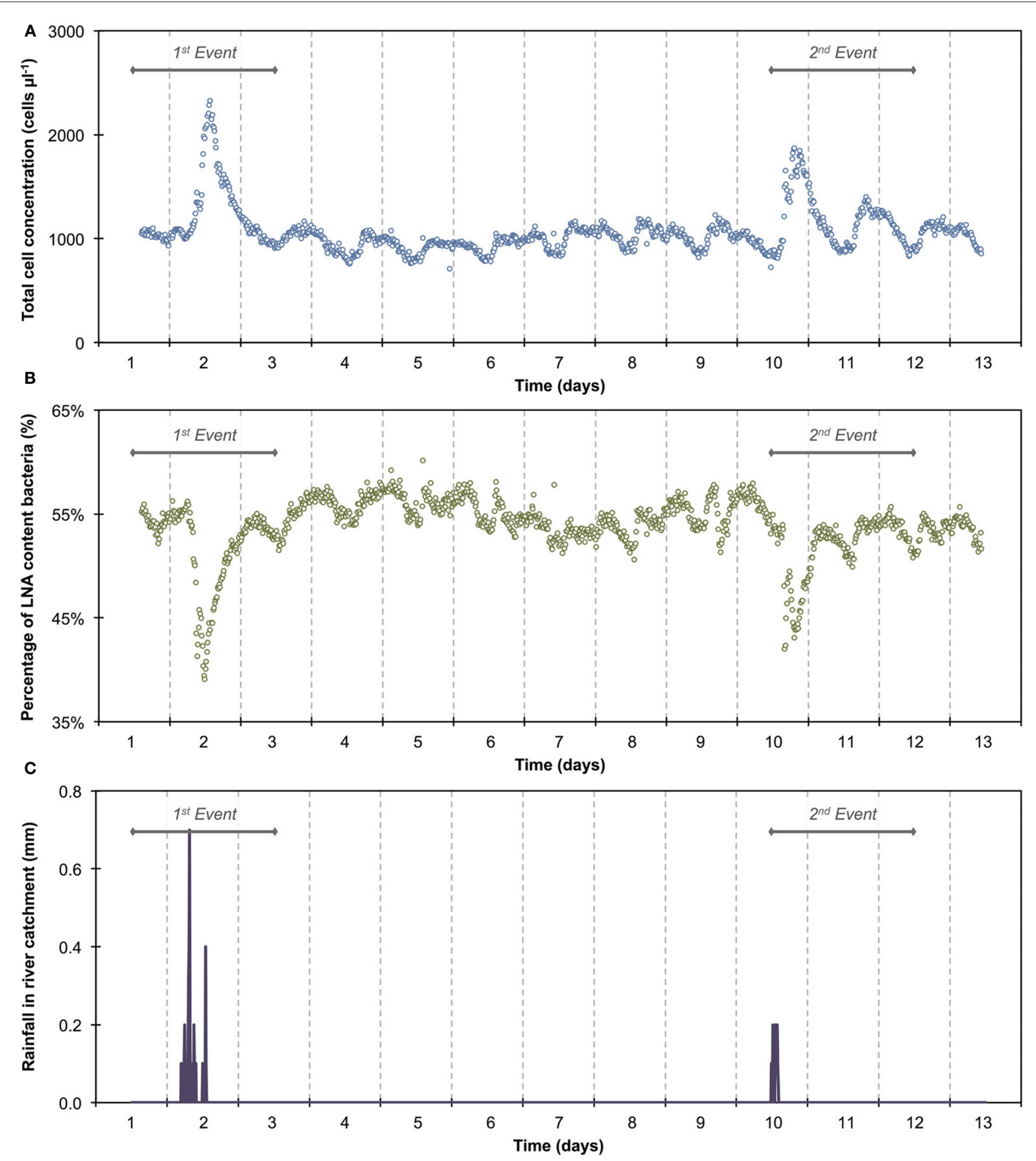

FIGURE 3 | Online FCM measurements of river water at 15-min intervals over 12 consecutive days, showing total cell concentrations (TCC) (A) and the percentage of LNA content bacteria (B) $(n=1104)$. Two distinct events can be seen on days $1-3$ and $10-12$, corresponding directly

interpretation of these dynamics is somewhat speculative. It was shown before that overnight stagnation in buildings leads to an increase in TCC and in the percentage of HNA content bacteria (hence by default a decrease in the percentage of LNA content with two rainfall events in the river catchment $\mathbf{( C )}$ and resulting in an increase in TCC and decrease in the percentage of LNA content bacteria. In addition, repeated daily patterns are visible, which are much smaller in magnitude than the two events.

bacteria), and also that flushing of taps leads to a decrease in both these parameters (Lautenschlager et al., 2010; Prest et al., 2013). This mirrors the general TCC and percentage of LNA content bacteria behavior seen in Figure 2. While the tap measured 


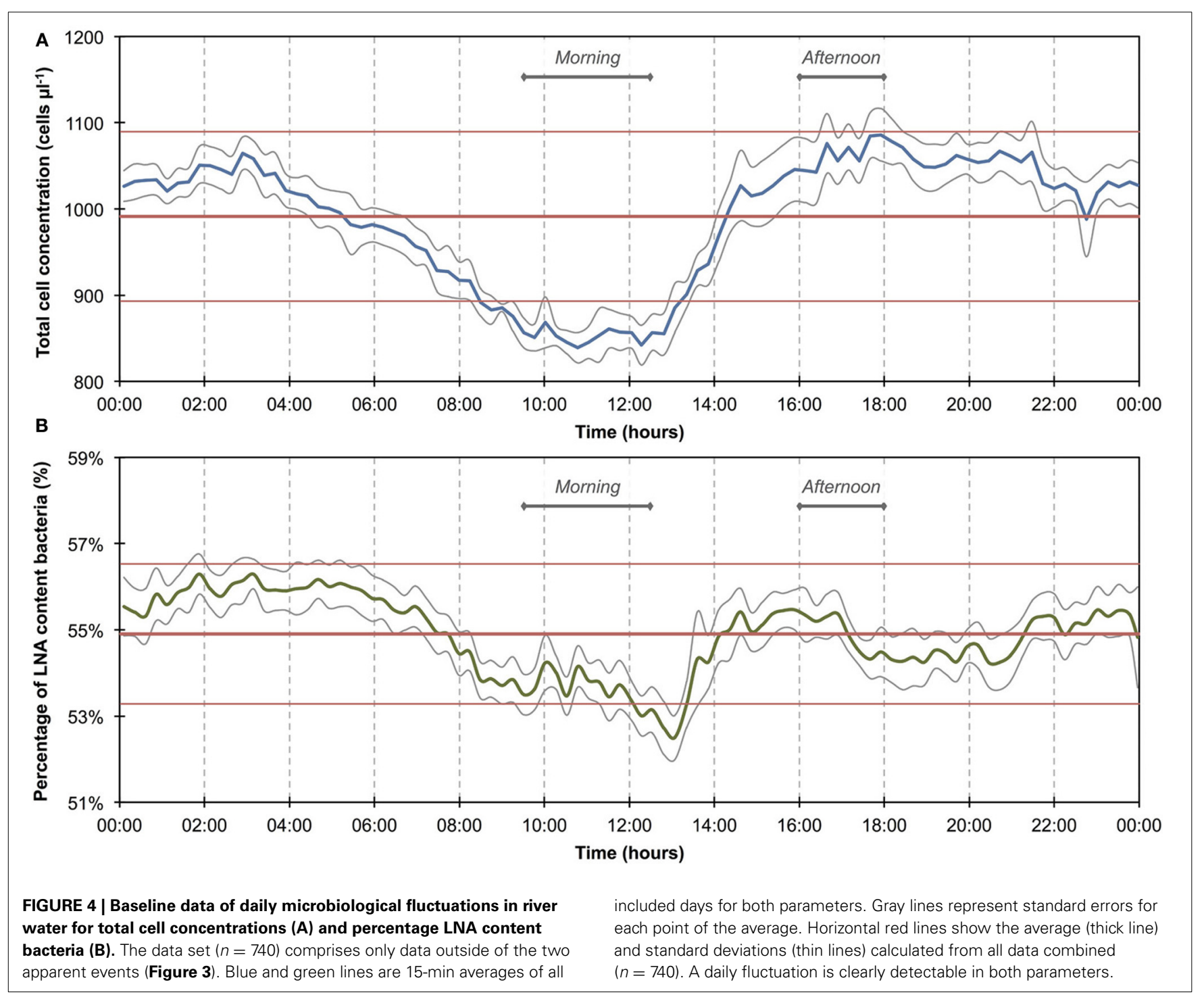

in the present study was continuously flowing, the remainder of the building was indeed subject to overnight stagnation. In fact, there is additional evidence that the FCM patterns followed localized water usage to some degree: (1) a dramatic decrease in TCC during the morning hours when most people normally arrive at work; (2) a peak during lunchtime which would correspond to a change in water usage; (3) the fact that weekends showed clearly dissimilar patterns to weekdays (Figure S5). All these fluctuations could relate to a combination of regrowth and changes in bacterial attachment/detachment due to changing hydraulic conditions on local or regional level (Lehtola et al., 2006). The dramatic increase in TCC on day 10 was unexpected. Discussions with the local building managers as well as the regional water authority yielded no additional information on any specific event during this period. The high frequency FCM data revealed previously unknown dynamics in the drinking water and suggests the investigation of detailed cause-effect relationships in future studies.

\section{BACTERIAL DYNAMICS IN RIVER WATER}

The average TCC measured in this study corresponded to many previous measurements of this particular river and is in the normal range of surface water (Wang et al., 2007). The data show an apparent daily fluctuation (Figures 3, 4) as well as two dramatic events linked to rainfall. Sensors for abiotic parameters also detected daily fluctuations and to some extent the rainfall events (Figure 5). The occurrence of diurnal fluctuations in the abiotic parameters (DO, EC, and $\mathrm{pH}$ ) has been described in detail for rivers, and is attributed to microbial photosynthetic and respiratory activities coupled to radiation and to subsequent changes in ion concentrations due to changes in precipitation and dissolution of calcite [for details see Vogt et al. (2010) and Hayashi et al. (2012)]. While we are not aware of any previous studies on fluctuations in the TCC at a similar time resolution, many of the processes that drive the abiotic fluctuations are likely to affect the microbiology as well. It is generally recognized that carbon compounds released by algae into the water during their daytime 


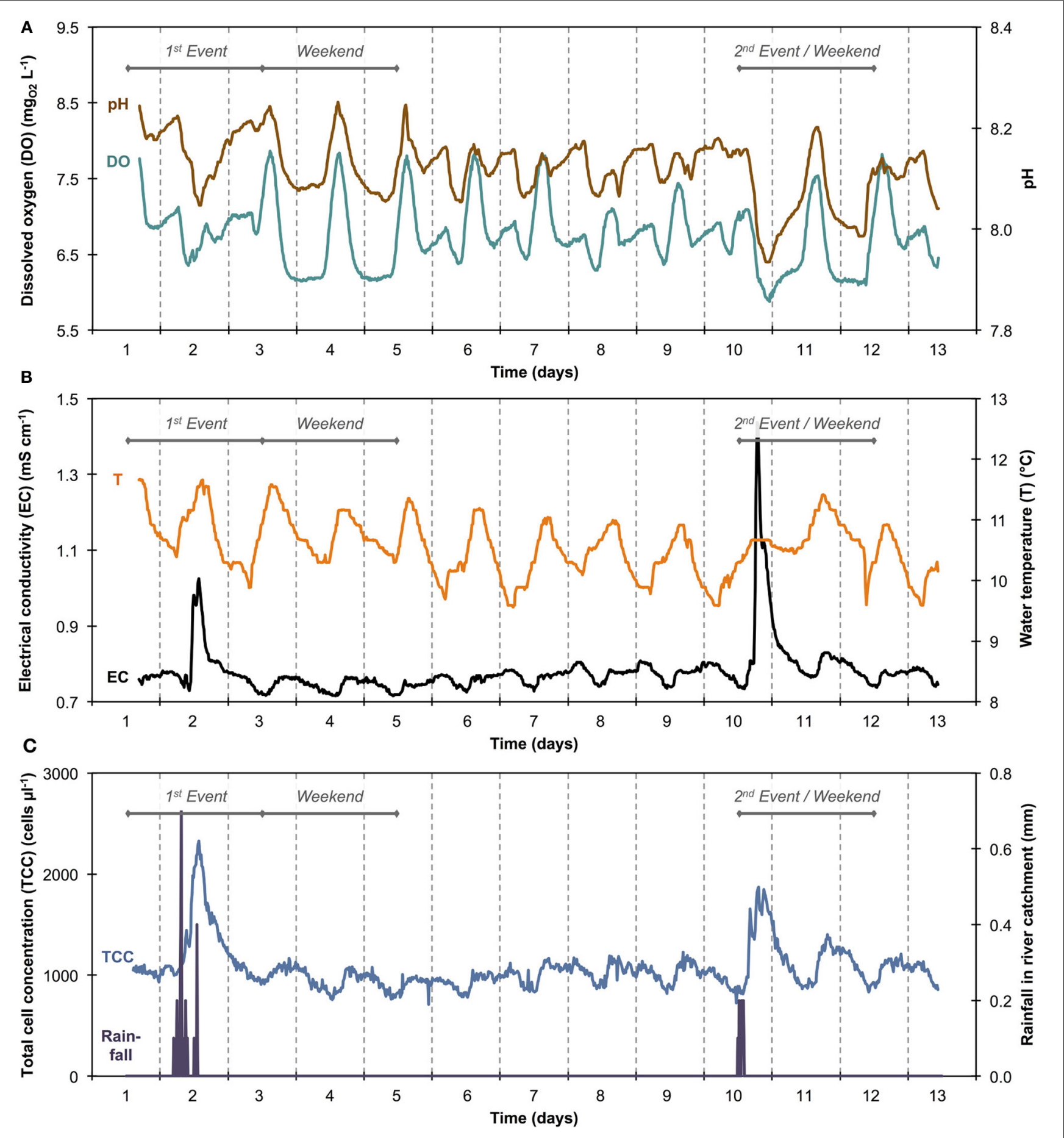

FIGURE 5 | Sensor data of river water for pH and dissolved oxygen (DO) (A) and electrical conductivity (EC) and temperature (T) (B). Total cell concentration (TCC) and rainfall data from Figure $\mathbf{3}$ are shown in simplified form (C) for easier and direct comparison with other sensor data.

activity serve as nutrients for bacteria and thus induce growth (Sundh and Bell, 1992). Algal mats and biofilms are present in this shallow river, which are likely to produce substantial amounts of carbon compounds. In addition, higher temperatures during the day may also favor increased bacterial growth. The measured increase in planktonic bacterial concentration is most likely due to increased detachment from river biofilms that could show increased growth for the reasons stated above. Figure S3A shows a clear positive correlation between conductivity and bacterial cell concentrations in dry periods and during rainfall events. Such correlations have been described in literature (Krishnamurti and Kate, 1951), although it is not clear whether the observed 


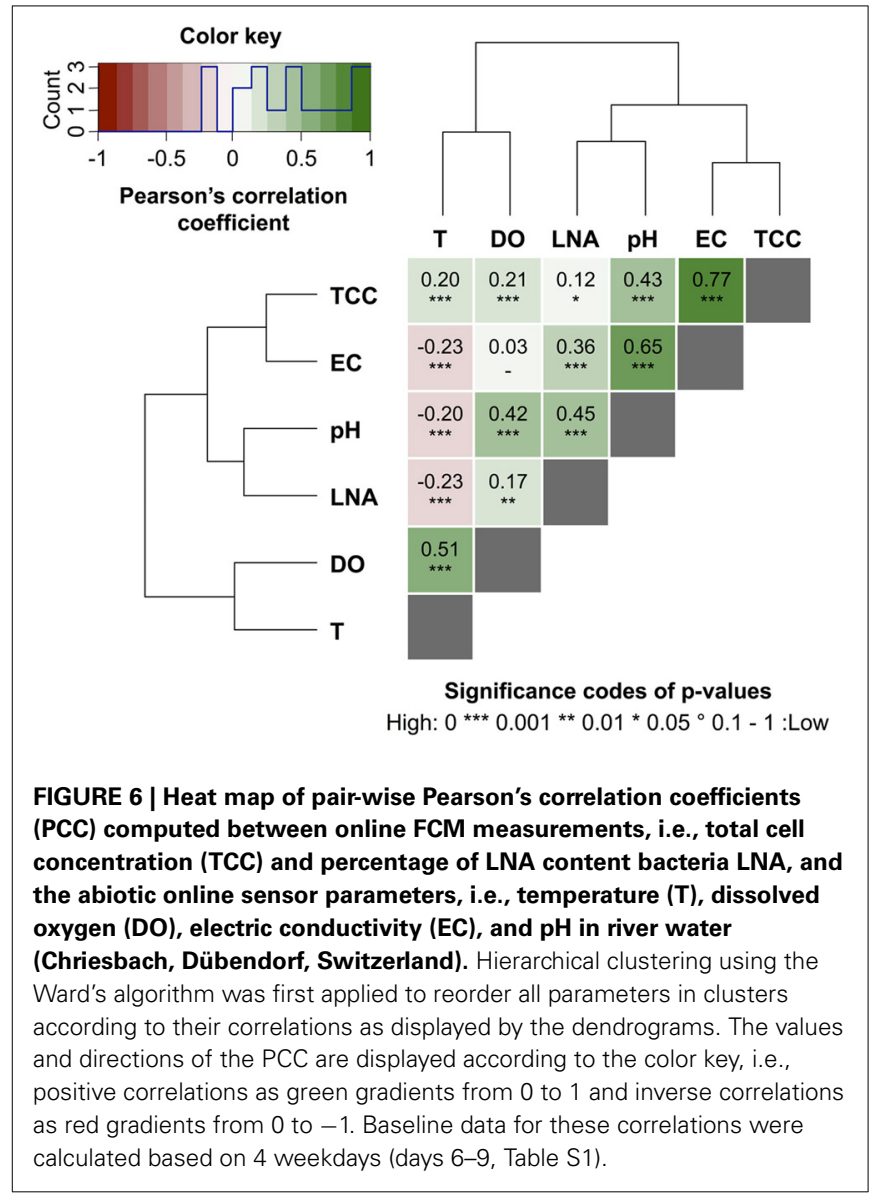

correlation in the present study was due to direct or indirect influences. Moreover, correlations during rainfall events are likely to have different causes from correlations during normal periods. During rainfall events, run-off from the surrounding catchment (including urban sewers) flows into the river and transports additional ions but also bacteria into the water body. This can explain the changes observed in the HNA/LNA pattern observed in Figure 3. However, during longer rainfall events, a dilution effect of relatively "pure" rainwater may occur after the initial peak due to run-off.

\section{CONSIDERATIONS ON INSTRUMENT ARTIFACTS}

Although the aim of the present study was not explicitly to assess cause-effect relationships in the studied ecosystems, one logic question is whether the cyclic patterns that we observed were actual events, or unexplained instrumental artifacts of the FCM or staining robot. We believe that the latter was not the case. Firstly, the changes in TCC in the river water do not follow the same pattern as the changes in the tap water (Figures 2, 4, Figure S6). Secondly, the relationship between the percentage of LNA content bacteria and TCC are different in the two ecosystems. Whereas in the tap water the percentage of LNA content bacteria tended to decrease with increasing TCC, the river water showed an opposite tendency (Figures 3, 4). Thirdly, the weekend did not show the same behavior in the tap water compared to weekdays (Figure S5). Finally, the sensors for abiotic parameters measuring the river water showed cyclic events as well (Figure 5 and Figure S4, discussed above). In addition, we did a comparison of automated FCM measurements with conventional manual FCM measurements, which showed similar data measured with both approaches (Figure S7). All these points strongly suggest that the changes/patterns were real and inherent to the ecosystems that were investigated.

\section{ADVANTAGES OF AUTOMATED ONLINE FCM MEASUREMENTS}

The experiments demonstrated several advantages of online FCM in monitoring:

(i) Long-term measurements allowed for the detection of stable phases, cyclic patterns and unexpected events, which would either be missed or incorrectly characterized by occasional grab sampling (Figures 1, 3). Events are not necessarily predictable in time and magnitude. It is thus ideal to use such long-term data to establish baselines that enable the identification and characterization of events as deviations from that baseline. As was shown in the basic data processing above, this can give a clear indication of critical time periods to be sampled/analyzed in more detail.

(ii) Frequent measurements allow for detailed analysis of events and fluctuations (Figures 2, 4). This is, for example, crucial when analyzing a flood event and comparing width and height of peaking curves of different parameters (Stadler et al., 2008; Butscher et al., 2011) or the relationship between different microbial parameters (e.g., TCC and the fluorescence fingerprint as highlighted for both experiments above).

(iii) Automated measurements allow for the recording of expected or unexpected events (e.g., snowmelt, rainfall) independent of the availability of personnel on location (Figure 3). Major events of interest may be rare or untimely and thus reliable automated systems increase the chance of measuring such events, even in remote locations (Stadler et al., 2008). In addition, automated systems free up human resources for other (non-automated) measurements in parallel during an event (e.g., conventional plating, ATP-measurements).

(iv) Online measurements allow real-time, in-situ monitoring of a variety of aquatic ecosystems that display temporal variability at different time scales, concentration ranges, and microbial community compositions. With both daily variations and specific events, two types of dynamics could be monitored in this study. Measurements were in the range of $10^{5}$ and $10^{6}$ cell $\mathrm{mL}^{-1}$ respectively and based on an earlier evaluation of FCM can be assumed to be accurate and sensitive (Hammes et al., 2012). The percentage of LNA content bacteria, as one simplistic form of FCM fingerprinting (SLMB, 2012; Prest et al., 2013), differed clearly between the two investigated ecosystems and within cycles and events. More advanced analysis of differences between systems and in time can be applied for example through extended fingerprinting methods (Koch et al., 2013a).

(v) Multi-parameter measurements, i.e., online FCM combined with other online sensors (e.g., temperature, conductivity), allow for robust differentiation between aquatic ecosystems 
and/or their respective conditions as well as identification of stability and deviations from it (Figures 2, 4). The combined biotic and abiotic data sets for the river water show interesting correlations and offer first insights into cause-and-effect relationships within the ecosystem (Section Correlations with Abiotic Sensor Data and Bacterial Dynamics in River Water, Figures 5, 6, and Figure S3).

\section{CHALLENGES WITH RESPECT TO IMPLEMENTATION AND METHOD ESTABLISHMENT}

FCM analysis of aquatic microorganisms has evolved considerably during the last decade (Hammes and Egli, 2010). However, despite the described advantages and the proof-of-concept data shown here (Figures 1, 3) and elsewhere (Hammes et al., 2012; Brognaux et al., 2013), routine application of online FCM is not yet reality. Several challenges with respect to implementation remain, and these differ for applications as a laboratory research tool and for application as a routine monitoring/sensor tool for water utilities.

Firstly, conventional FCM hardware is still relatively expensive (20-50 kUSD) today, although it is expected to decrease in price in the future. For the system described herein, we estimated operating costs of approximately 0.1 USD per measurement (thus $<10.0$ USD per day), excluding instrument purchase (Table S8). The automated staining robot used herein was a prototype that is not currently commercially available, but construction of similar systems is clearly feasible (Hammes et al., 2012; Brognaux et al., 2013).

Secondly, the applicability of the automated system to different aquatic ecosystems is limited with respect to cell concentrations and background levels in the samples of interest. Commercially available bench top flow cytometers typically measure in a range of $10^{3}-10^{6}$ cells $\mathrm{mL}^{-1}$ (Hammes et al., 2008, 2012), which is suitable for a variety of aquatic ecosystems such as groundwater, tap water, and rivers and lakes (Wang et al., 2007). However, for more concentrated samples [e.g., in bioreactors or activated sludge settings (Koch et al., 2013b)], extensive dilution techniques would need to be included (Abu-Absi et al., 2003; Broger et al., 2011). This potentially adds costs and measurement error to the system. In general, none-bacterial particles may cause clogging of instruments and overlaying of background and bacterial signals (Hammes and Egli, 2010). Although we experienced no problems in the current study, this may be of particular importance in event monitoring, where for example rain events may cause substantial turbidity in the sampled water (Pronk et al., 2006).

Thirdly, monitoring with automated online FCM generates data sets comprising hundreds-to-thousands of data points for several parameters (e.g., TCC, green/red fluorescence intensity, forward/sideward scatter). It has previously been shown that standardization throughout the FCM analysis train (including data processing) is essential for high quality data (SLMB, 2012; Prest et al., 2013). Efficient, standardized data processing and analysis will be crucial to make optimal use of the newly available richness in microbial data. Our data sets were already so large that we required custom software for processing and extension of this can include advanced fingerprinting methods (Koch et al., 2013a; Prest et al., 2013) and time series analysis (Pomati et al., 2013). Through the quantification of microbial variability (e.g., temporal, geographical), relationships between observed patterns and potential drivers (e.g., biofilm attachment and detachment, contamination, mixing, treatment methods, temperature) can be tested systematically.

\section{APPLICATIONS}

We envisage a broad range of future applications of fully automated online FCM. Firstly, in treatment process optimization but also routine operation of drinking water/wastewater treatment plants, online microbial monitoring can help to investigate different modes of operation or the influence of diurnal process changes or changing raw water quality (Hammes et al., 2008; Vital et al., 2012). Similar applications can be of value in related industries such as bottled water production, beer brewing or the production of ultra-pure water. Secondly, in environmental aquatic ecosystems, interactions between abiotic and biotic factors both for regular and event-based dynamics can be studied with considerable detail using this approach (Pronk et al., 2006; Stadler et al., 2010; Butscher et al., 2011). Thirdly, automated measuring of microbial parameters renders event monitoring for quantitative microbial risk assessment considerably much more feasible (Signor and Ashbolt, 2006). Finally, a large variety of laboratory-based research can benefit from high temporal resolution and automation. Straightforward examples are experiments on bacterial batch growth or continuous cultures under different conditions (e.g., different media, inhibiting agents) or experiments looking at different types and intensities of disinfection (Arnoldini et al., 2013).

\section{CONCLUSIONS}

Our results demonstrate that automated in-situ FCM analysis is feasible for the investigation of dynamic aquatic ecosystems at high temporal resolution during multiple days.

The resulting large sets of reliable, quantitative microbiological data considerably improved characterization of dynamic fluctuations, which in turn enhances our understanding of complex engineered and environmental ecosystems.

The data revealed both daily fluctuations and specific events in the drinking water and river water ecosystems, suggesting that temporal variations should be carefully taken into consideration during sampling and analysis of aquatic ecosystems.

Automated FCM allows for the combination of microbial data at high temporal resolution with conventional online parameters, thus expanding the horizon toward a complete sensing approach.

\section{AUTHOR CONTRIBUTIONS}

Hardware/software development: Bradley E. Kratochvil, Mathias S. Weyland. Experimental design: Michael D. Besmer, Jürg A. Sigrist, Frederik Hammes. Research: Michael D. Besmer, David G. Weissbrodt, Jürg A. Sigrist, Frederik Hammes. Data analysis: Michael D. Besmer, David G. Weissbrodt, Mathias S. Weyland, Frederik Hammes. Writing/editing: Michael D. Besmer, David G. Weissbrodt, Bradley E. Kratochvil, Jürg A. Sigrist, Mathias S. Weyland, Frederik Hammes. 


\section{ACKNOWLEDGMENTS}

The authors acknowledge the financial support from the Canton Basel-Landschaft, Switzerland in the framework of the project "Regionale Wasserversorgung Basel-Landschaft 21" as well as internal Eawag Discretionary Funding. The authors thank Beat Schwarzenbach from Empa Dübendorf for providing rainfall data from the NABEL project (FOEN and EMPA). The authors thank Dr. Francesco Pomati for advice on data analysis.

\section{SUPPLEMENTARY MATERIAL}

The Supplementary Material for this article can be found online at: http://www.frontiersin.org/journal/10.3389/fmicb. 2014.00265/abstract

\section{REFERENCES}

Abu-Absi, N. R., Zamamiri, A., Kacmar, J., Balogh, S. J., and Srienc, F. (2003). Automated flow cytometry for acquisition of time-dependent population data. Cytometry A. 51, 87-96. doi: 10.1002/cyto.a.10016

Arnoldini, M., Heck, T., Blanco-Fernandez, A., and Hammes, F. (2013). Monitoring of dynamic microbiological processes using real-time flow cytometry. PLoS ONE 8:117. doi: 10.1371/journal.pone.0080117

Borcard, D., Gillet, F. O., and Legendre, P. (2011). Numerical Ecology with R. New York; London: Springer.

Broger, T., Odermatt, R. P., Huber, P., and Sonnleitner, B. (2011). Real-time online flow cytometry for bioprocess monitoring. J. Biotechnol. 154, 240-247. doi: 10.1016/j.jbiotec.2011.05.003

Brognaux, A., Han, S., Sorensen, S., Lebeau, F., Thonart, P., and Delvigne, F. (2013). A low-cost, multiplexable, automated flow cytometry procedure for the characterization of microbial stress dynamics in bioreactors. Microb. Cell Fact. 12:100. doi: 10.1186/1475-2859-12-100

Butscher, C., Auckenthaler, A., Scheidler, S., and Huggenberger, P. (2011). Validation of a numerical indicator of microbial contamination for karst springs. Ground Water 49, 66-76. doi: 10.1111/j.1745-6584.2010. 00687.x

Czechowska, K., Johnson, D. R., and van der Meer, J. R. (2008). Use of flow cytometric methods for single-cell analysis in environmental microbiology. Curr. Opin. Microbiol. 11, 205-212. doi: 10.1016/j.mib.2008.04.006

Day, A. (2007). heatmap.plus: Heatmaps with More Sensible Behavior. R Package Version 1.3. Vienna: R Foundation for Statistical Computing. Available online at: http://cran.r-project.org/web/packages/heatmap.plus/index.html

Dubelaar, G. B. J., Gerritzen, P. L., Beeker, A. E. R., Jonker, R. R., and Tangen, K. (1999). Design and first results of CytoBuoy: a wireless flow cytometer for in situ analysis of marine and fresh waters. Cytometry 37, 247-254. doi: 10.1002/(SICI)1097-0320(19991201)37:4<247::AID-CYTO1> 3.0.CO;2-9

Foladori, P., Bruni, L., Tamburini, S., and Ziglio, G. (2010). Direct quantification of bacterial biomass in influent, effluent and activated sludge of wastewater treatment plants by using flow cytometry. Water Res. 44, 3807-3818. doi: 10.1016/j.watres.2010.04.027

Gregori, G., Citterio, S., Ghiani, A., Labra, M., Sgorbati, S., Brown, S., et al. (2001). Resolution of viable and membrane-compromised bacteria in freshwater and marine waters based on analytical flow cytometry and nucleic acid double staining. Appl. Environ. Microbiol. 67, 4662-4670. doi: 10.1128/AEM.67.10.46624670.2001

Guadayol, O., Peters, F., Marrase, C., Gasol, J. M., Roldan, C., Berdalet, E., et al. (2009). Episodic meteorological and nutrient-load events as drivers of coastal planktonic ecosystem dynamics: a time-series analysis. Mar. Ecol. Prog. Ser. 381, 139-155. doi: 10.3354/meps07939

Gunther, S., Koch, C., Hubschmann, T., Roske, I., Muller, R. A., Bley, T., et al. (2012). Correlation of community dynamics and process parameters as a tool for the prediction of the stability of wastewater treatment. Environ. Sci. Technol. 46, 84-92. doi: 10.1021/es2010682

Hammes, F., Berney, M., Wang, Y. Y., Vital, M., Koster, O., and Egli, T. (2008). Flow-cytometric total bacterial cell counts as a descriptive microbiological parameter for drinking water treatment processes. Water Res. 42, 269-277. doi: 10.1016/j.watres.2007.07.009
Hammes, F., Broger, T., Weilenmann, H. U., Vital, M., Helbing, J., Bosshart, U., et al. (2012). Development and laboratory-scale testing of a fully automated online flow cytometer for drinking water analysis. Cytometry A. 81, 508-516. doi: 10.1002/cyto.a.22048

Hammes, F., and Egli, T. (2010). Cytometric methods for measuring bacteria in water: advantages, pitfalls and applications. Anal. Bioanal. Chem. 397, 1083-1095. doi: 10.1007/s00216-010-3646-3

Hayashi, M., Vogt, T., Machler, L., and Schirmer, M. (2012). Diurnal fluctuations of electrical conductivity in a pre-alpine river: Effects of photosynthesis and groundwater exchange. J. Hydrol. 450, 93-104. doi: 10.1016/j.jhydrol.2012.05.020

Hwang, C., Ling, F. Q., Andersen, G. L., Lechevallier, M. W., and Liu, W. T. (2012). Microbial community dynamics of an urban drinking water distribution system subjected to phases of chloramination and chlorination treatments. Appl. Environ. Microbiol. 78, 7856-7865. doi: 10.1128/AEM.01892-12

Koch, C., Fetzer, I., Harms, H., and Muller, S. (2013a). CHIC-an automated approach for the detection of dynamic variations in complex microbial communities. Cytometry A. 83, 561-567. doi: 10.1002/cyto.a.22286

Koch, C., Fetzer, I., Schmidt, T., Harms, H., and Muller, S. (2013b). Monitoring functions in managed microbial systems by cytometric bar coding. Environ. Sci. Technol. 47, 1753-1760. doi: 10.1021/es3041048

Krishnamurti, K., and Kate, S. R. (1951). Changes in electrical conductivity during bacterial growth. Nature 168, 170-170. doi: 10.1038/ 168170b0

Lautenschlager, K., Boon, N., Wang, Y. Y., Egli, T., and Hammes, F. (2010). Overnight stagnation of drinking water in household taps induces microbial growth and changes in community composition. Water Res. 44, 4868-4877. doi: 10.1016/j.watres.2010.07.032

Legendre, L., Courties, C., and Troussellier, M. (2001). Flow cytometry in oceanography 1989-1999: environmental challenges and research trends. Cytometry 44, 164-172. doi: 10.1002/1097-0320(20010701)44:3<164::AIDCYTO1108>3.0.CO;2-6

Lehtola, M. J., Laxander, M., Miettinen, I. T., Hirvonen, A., Vartiainen, T., and Martikainen, P. J. (2006). The effects of changing water flow velocity on the formation of biofilms and water quality in pilot distribution system consisting of copper or polyethylene pipes. Water Res. 40, 2151-2160. doi: 10.1016/j.watres.2006.04.010

Lindberg, W., Ruzicka, J., and Christian, G. D. (1993). Flow-injection cytometrya new approach for sample and solution handling in flow-cytometry. Cytometry 14, 230-236. doi: 10.1002/cyto.990140217

Oksanen, J., Kindt, R., Legendre, P., O’Hara, B., Simpson, G. L., Solymos, P., et al. (2009). Vegan: Community Ecology Package. $R$ Package Version 1.154. Vienna: R Foundation for Statistical Computing. Available online at: http://CRAN.R-project.org/package=vegan

Omann, G. M., Coppersmith, W., Finney, D. A., and Sklar, L. A. (1985). A convenient online device for reagent addition, sample mixing, and temperature control of cell-suspensions in flow-cytometry. Cytometry 6, 69-73. doi: 10.1002/cyto.990060113

Ploner, A. (2011). Heatplus: Heatmaps with Row and/or Column Covariates and Colored Clusters. R Package Version 2.1.0. Vienna: R Foundation for Statistical Computing. Available online at: http://www.bioconductor.org/packages/2.10/ bioc/html/Heatplus.html

Pomati, F., Kraft, N. J. B., Posch, T., Eugster, B., Jokela, J., and Ibelings, B. W. (2013). Individual cell based traits obtained by scanning flow-cytometry show selection by biotic and abiotic environmental factors during a phytoplankton spring bloom. PLoS ONE 8:e71677. doi: 10.1371/journal.pone.0071677

Porter, J., Deere, D., Hardman, M., Edwards, C., and Pickup, R. (1997). Go with the flow-use of flow cytometry in environmental microbiology. FEMS Microbiol. Ecol. 24, 93-101. doi: 10.1016/S0168-6496(97)00038-X

Prest, E. I., Hammes, F., Kötzsch, S., Van Loosdrecht, M. C. M., and Vrouwenvelder, J. S. (2013). Monitoring microbiological changes in drinking water systems using a fast and reproducible flow cytometric method. Water Res. 47, 7131-7142. doi: 10.1016/j.watres.2013.07.051

Pronk, M., Goldscheider, N., and Zopfi, J. (2006). Dynamics and interaction of organic carbon, turbidity and bacteria in a karst aquifer system. Hydrogeol. J. 14, 473-484. doi: 10.1007/s10040-005-0454-5

Read, S., Marzorati, M., Guimaraes, B. C. M., and Boon, N. (2011). Microbial Resource Management revisited: successful parameters and new concepts. Appl. Microbiol. Biotechnol. 90, 861-871. doi: 10.1007/s00253-011-3223-5 
R Development Core Team (2008). R: A Language and Environment for Statistical Computing. Vienna: R Foundation for Statistical Computing. Available online at: http://cran.r-project.org/

Sheikh, Q. I., Boxall, J. B., and Biggs, C. A. (2012). "A review of potential culture independent biological detection methods for the water industry-challenges of moving beyond the research lab," in The Significance of Faecal Indicators in Water: A Global Perspective, eds D. Kay and C. Fricker (Cambridge: RSC Publishing), 131-144. doi: 10.1039/9781849735421-00131

Signor, R. S., and Ashbolt, N. J. (2006). Pathogen monitoring offers questionable protection against drinking-water risks: a QMRA (Quantitative Microbial Risk Analysis) approach to assess management strategies. Water Sci. Technol. 54, 261-268. doi: 10.2166/wst.2006.478

SLMB. (2012). "Method 333.1: determining the total cell count and ratios of high and low nucleic acid content cells in freshwater using flow cytometry," in Schweizerisches Lebensmittelhandbuch (Bern: Federal Office for Public Health).

Smeets, P. W. M. H., Rietveld, L. C., Van Dijk, J. C., and Medema, G. J. (2010). Practical applications of quantitative microbial risk assessment (QMRA) for water safety plans. Water Sci. Technol. 61, 1561-1568. doi: 10.2166/wst. 2010.839

Stadler, H., Klock, E., Skritek, P., Mach, R. L., Zerobin, W., and Farnleitner, A. H. (2010). The spectral absorption coefficient at $254 \mathrm{~nm}$ as a real-time early warning proxy for detecting faecal pollution events at alpine karst water resources. Water Sci. Technol. 62, 1898-1906. doi: 10.2166/wst.2010.500

Stadler, H., Skritek, P., Sommer, R., Mach, R. L., Zerobin, W., and Farnleitner, A. H. (2008). Microbiological monitoring and automated event sampling at karst springs using LEO-satellites. Water Sci. Technol. 58, 899-909. doi: $10.2166 /$ wst. 2008.442

Sundh, I., and Bell, R. T. (1992). Extracellular dissolved organic-carbon released from phytoplankton as a source of carbon for heterotrophic bacteria in lakes of different humic content. Hydrobiologia 229, 93-106. doi: 10.1007/BF000 06993

Thyssen, M., Tarran, G. A., Zubkov, M. V., Holland, R. J., Gregori, G., Burkill, P. H., et al. (2008). The emergence of automated high-frequency flow cytometry: revealing temporal and spatial phytoplankton variability. J. Plankton Res. 30, 333-343. doi: 10.1093/plankt/fbn005

Van Den Hoven, T., Kazner, C., Project Techneau (European Commission), and European Commission. (2009). TECHNEAU: Safe Drinking Water from Source to Tap State of the Art And Perspectives. London; New York: IWA Pub.

Vital, M., Dignum, M., Magic-Knezeu, A., Ross, P., Rietueld, L., and Hammes, F. (2012). Flow cytometry and adenosine tri-phosphate analysis: alternative possibilities to evaluate major bacteriological changes in drinking water treatment and distribution systems. Water Res. 46, 4665-4676. doi: 10.1016/j.watres.2012.06.010

Vives-Rego, J., Lebaron, P., and Nebe-Von Caron, G. (2000). Current and future applications of flow cytometry in aquatic microbiology. FEMS Microbiol. Rev. 24, 429-448. doi: 10.1111/j.1574-6976.2000.tb00549.x

Vogt, T., Hoehn, E., Schneider, P., Freund, A., Schirmer, M., and Cirpka, O. A. (2010). Fluctuations of electrical conductivity as a natural tracer for bank filtration in a losing stream. Adv. Water Resour. 33, 1296-1308. doi: 10.1016/j.advwatres.2010.02.007

Wang, Y., Hammes, F., Boon, N., and Egli, T. (2007). Quantification of the filterability of freshwater bacteria through $0.45,0.22$, and $0.1 \mathrm{mu}$ m pore size filters and shape-dependent enrichment of filterable bacterial communities. Environ. Sci. Technol. 41, 7080-7086. doi: 10.1021/es0707198

Weissbrodt, D. G., Shani, N., and Holliger, C. (2014). Linking bacterial population dynamics and nutrient removal in the granular sludge biofilm ecosystem engineered for wastewater treatment. FEMS Microbiol. Ecol. doi: 10.1111/1574-6941. 12326. [Epub ahead of print].

World Health Organization. (2011). Guidelines for Drinking-Water Quality. Geneva: World Health Organization.

Zhao, R., Natarajan, A., and Srienc, F. (1999). A flow injection flow cytometry system for on-line monitoring of bioreactors. Biotechnol. Bioeng. 62, 609-617. doi: 10.1002/(SICI)1097-0290(19990305)62:5<609::AID-BIT13>3.0.CO;2-C

Conflict of Interest Statement: The authors declare that the research was conducted in the absence of any commercial or financial relationships that could be construed as a potential conflict of interest.

Received: 20 February 2014; paper pending published:09 April 2014; accepted: 15 May 2014; published online: 02 June 2014.

Citation: Besmer MD, Weissbrodt DG, Kratochvil BE, Sigrist JA, Weyland MS and Hammes $F$ (2014) The feasibility of automated online flow cytometry for in-situ monitoring of microbial dynamics in aquatic ecosystems. Front. Microbiol. 5:265. doi: 10.3389/fmicb.2014.00265

This article was submitted to Systems Microbiology, a section of the journal Frontiers in Microbiology.

Copyright (c) 2014 Besmer, Weissbrodt, Kratochvil, Sigrist, Weyland and Hammes. This is an open-access article distributed under the terms of the Creative Commons Attribution License (CC BY). The use, distribution or reproduction in other forums is permitted, provided the original author(s) or licensor are credited and that the original publication in this journal is cited, in accordance with accepted academic practice. No use, distribution or reproduction is permitted which does not comply with these terms. 